\title{
Bounding the parameters of linear systems with stability constraints
}

\author{
V. Cerone, D. Piga, D. Regruto
}

\begin{abstract}
Identification of linear systems, a priori known to be stable, from input output measurements corrupted by bounded noise is considered in the paper. A formal definition of the feasible parameter set is provided, taking explicitly into account prior information on system stability. On the basis of a detailed analysis of the geometrical structure of the feasible set, convex relaxation techniques are presented to solve nonconvex optimization problems arising in the computation of the parameters uncertainty intervals. Properties of the computed relaxed bounds are discussed. A simulated example is presented to show the effectiveness of the proposed technique.

Index Terms-Set-membership identification, LMI relaxation, stability constraints
\end{abstract}

\section{INTRODUCTION}

According to Ljung [1], any system identification procedure involves three basic ingredients: a set of inputoutput measurements, a set of candidate models and the identification method, which can roughly be described as a rule to select a model among the candidate ones on the basis of the measured data and a proper model quality assessment criterion. The choice of the set of candidate models, sometimes called model structure, is the most critical step since it strongly relies on the available a priori information: practical experience, physical insights and engineering intuitions play here a crucial role. Restricting our attention to the case of Linear Time Invariant (LTI) systems, Bounded Input Bounded Output (BIBO) stability is perhaps the most common assumption when open-loop identification procedures are of interest. Indeed, when this hypothesis is not satisfied, open-loop experiments cannot be performed in practice. Although many times the system to be identified is surely known to be stable, most of the identification techniques do not exploit such a prior information in the definition of the assumed model structure, since formal inclusion of mathematical constraints related to stability makes the estimation problem difficult to be solved. As a result, the identification procedure may give rise to inaccurate models and even instability may arise, especially in the presence of shortage of data, modeling error and measurement noise. Only few contributions are available in the literature addressing the problem of how taking into account prior information about system stability. In paper [2] Söderström and Stoica consider the identification of input-output linear dynamics systems described by difference equations; through a simple counterexample, they show that application of the Least Squares (LS) method may lead to unstable models

The authors are with the Dipartimento di Automatica e Informatica, Politecnico di Torino, corso Duca degli Abruzzi 24, 10129 Torino, Italy; e-mail: vito.cerone@polito.it, dario.piga@polito.it, diego.regruto@polito.it; Tel: +39-011-564 7064; Fax: +39-011-564 7198 when certain conditions in terms of signal-to-noise ratio are satisfied. A sufficient condition to ensure stability of dynamic models obtained by LS identification is provided in [3] where the input signal is constrained to be an autoregressive process of a given degree. Tugnait and Tontiruttananon in [4] provide a frequency domain solution to LS identification of a stable system in presence of undermodeling. They present an approach that applies when the input signal is a zeromean stationary process with sufficiently high persistency of excitation order. A stable output error identification scheme is presented in [5] for the case of all-pole systems and periodic excitation signals, while a procedure to include prior information on BIBO stability in the context of the kernelbased nonparametric identification is discussed in [6]. As far as subspace identification is concerned, some different approaches have been introduced in the last decade to enforce stability. The interested reader can refer to [7] and the references therein for a thorough review on the subject. The most recent and effective among such approaches is the one presented by Bernstein and Lacy in [7] where prior information on asymptotic stability is directly taken into account computing the LS estimate through the solution of a proper convex optimization problem.

A common assumption in system identification is that the measurement error is statistically described. However, when uncertainties are known to belong to a given set, a setmembership characterization of measurement errors should be preferred to the stochastic description. Some examples include mechanical tolerances, analog-to-digital converter quantization errors, systematic and class errors in measurement equipments. In this context, all parameters consistent with the measurements, the error bounds and the assumed model structure, are feasible solutions of the identification problem. The interested reader can find further details on this approach in a number of survey papers (see, e.g., [8], [9]), in the book edited by Milanese et al. [10], and the special issues edited by Norton [11], [12].

In this work, we consider the identification of SISO discrete-time linear systems that are a priori known to be stable. The aim of the paper is to compute bounds on the system parameters when both the input and output data are corrupted by bounded noise. To the authors' best knowledge, no contribution can be found in the literature on the identification problem addressed in this paper.

The note is organized as follows. Section II is devoted to the formulation of the problem. First a formal definition of the feasible parameter set is provided taking explicitly into account prior information on system stability. Then, computation of the parameter uncertainty intervals is formulated 


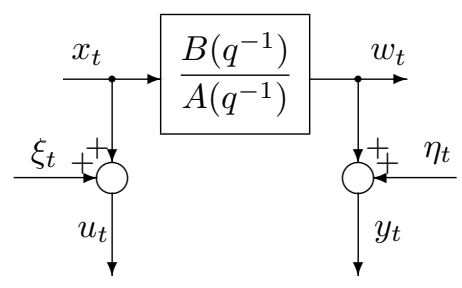

Fig. 1. Errors-in-variable setup for dynamic linear system.

in terms of nonlinear nonconvex optimization. A detailed analysis of the geometrical structure of the defined feasible parameter set is presented in Section III. On the basis of this analysis, suitable convex relaxation techniques are discussed to solve the nonconvex optimization problems presented in Section II. In Section IV, accuracy and convergency properties of the relaxed bounds computed in Section III are discussed. A simulated example is reported in Section $\mathrm{V}$ in order to highlight the improvement obtained in the computation of the parameter bounds when prior information on stability are explicitly taken into account.

\section{PROBLEM FORMULATION}

Consider the Single Input Single Output (SISO) LinearTime-Invariant (LTI) system depicted in Fig. 1. The linear dynamic system is modeled by a discrete time system that transforms the noise-free input sequence $x_{t}$ into the noisefree output $w_{t}$ according to the difference equation

$$
A\left(q^{-1}\right) w_{t}=B\left(q^{-1}\right) x_{t},
$$

where $A(\cdot)$ and $B(\cdot)$ are polynomials in the backward shift operator $q^{-1}\left(q^{-1} w_{t}=w_{t-1}\right)$ :

$$
\begin{aligned}
& A\left(q^{-1}\right)=1+a_{1} q^{-1}+\ldots+a_{n a} q^{-n a} \\
& B\left(q^{-1}\right)=b_{0}+b_{1} q^{-1}+\ldots+b_{n b} q^{-n b}
\end{aligned}
$$

Let $u_{t}$ and $y_{t}$ be the noise-corrupted input and output measured sequence respectively

$$
\begin{aligned}
& u_{t}=x_{t}+\xi_{t} \\
& y_{t}=w_{t}+\eta_{t} .
\end{aligned}
$$

Measurement uncertainties $\xi_{t}$ and $\eta_{t}$ are assumed to range within given bounds $\Delta \xi_{t}$ and $\Delta \eta_{t}$ respectively, that is:

$$
\begin{aligned}
& \left|\xi_{t}\right| \leq \Delta \xi_{t} \\
& \left|\eta_{t}\right| \leq \Delta \eta_{t}
\end{aligned}
$$

The unknown parameter vector $\theta \in \mathbb{R}^{p}$ to be estimated is defined as

$$
\theta^{\mathrm{T}}=\left[\begin{array}{lllllll}
a_{1} & \ldots & a_{n a} & b_{0} & b_{1} & \ldots & b_{n b}
\end{array}\right]
$$

where $n a+n b+1=p$.

In the set-membership context, all parameter vectors belonging to the feasible parameter set (FPS), i.e. parameters consistent with the measurements, the error bounds and the assumed model structure, are feasible solutions of the identification problem. Given $N$ samples of the signals $u_{t}$ and $y_{t}$, the feasible parameter set $\mathcal{D}_{\theta}$ of the linear system described by equations (1) - (7) is defined as

$$
\begin{gathered}
\mathcal{D}_{\theta}=\left\{\theta \in \mathbb{R}^{p}: A\left(q^{-1}\right)\left(y_{t}-\eta_{t}\right)=B\left(q^{-1}\right)\left(u_{t}-\xi_{t}\right),\right. \\
\left.\left|\xi_{t}\right| \leq \Delta \xi_{t}, \quad\left|\eta_{t}\right| \leq \Delta \eta_{t} ; t=1, \ldots, N\right\} .
\end{gathered}
$$

The exact feasible parameter region $\mathcal{D}_{\theta}$ is a nonconvex set described by nonlinear inequalities, whose shape may become fairly complex for increasing values of $N$. As a consequence, parameters bounds might not be easily computed on the basis of $\mathcal{D}_{\theta}$ [13]. In order to overcome such a problem, the following outer approximation $\mathcal{D}_{\theta}^{\prime}$ of the exact FPS $\mathcal{D}_{\theta}$, i.e. $\mathcal{D}_{\theta}^{\prime} \supset \mathcal{D}_{\theta}$ has been proposed in [14], [15]:

$$
\begin{aligned}
\mathcal{D}_{\theta}^{\prime}= & \left\{\theta \in \mathbb{R}^{p}:\left(\phi_{t}-\Delta \phi_{t}\right) \theta \leq y_{t}+\Delta \eta_{t},\right. \\
& \left.\left(\phi_{t}+\Delta \phi_{t}\right) \theta \geq y_{t}-\Delta \eta_{t} ; \quad t=1, \ldots, N\right\}
\end{aligned}
$$

where $\phi_{t}$ is the regression vector:

$$
\phi_{t}^{\mathrm{T}}=\left[\begin{array}{lllllll}
-y_{t-1} & \ldots & -y_{t-n a} & u_{t} & u_{t-1} & \ldots & u_{t-n b}
\end{array}\right]
$$

and

$$
\begin{aligned}
\Delta \phi_{t}^{\mathrm{T}}= & {\left[\Delta \eta_{t-1} \operatorname{sgn}\left(a_{1}\right) \ldots \Delta \eta_{t-n a} \operatorname{sgn}\left(a_{n a}\right) \Delta \xi_{t} \operatorname{sgn}\left(b_{0}\right)\right.} \\
& \left.\Delta \xi_{t-1} \operatorname{sgn}\left(b_{1}\right) \ldots \Delta \xi_{t-n b} \operatorname{sgn}\left(b_{n b}\right)\right] .
\end{aligned}
$$

$\mathcal{D}_{\theta}^{\prime}$ is the union of at most $2^{p}$ convex regions in $\mathbb{R}^{p}$, i.e.

$$
\mathcal{D}_{\theta}^{\prime}=\bigcup_{i=1}^{2^{p}} \mathcal{D}_{\theta i}^{\prime}
$$

where each $\mathcal{D}_{\theta i}^{\prime}$ is a polytope defined by $2 N+p$ linear constraints obtained through the intersection of $\mathcal{D}_{\theta}^{\prime}$ with the $i$-th orthant of the parameter space $\mathbb{R}^{p}$.

On the basis of the set $\mathcal{D}_{\theta}^{\prime}$, lower and upper bounds $\theta_{j}$ and $\overline{\theta_{j}}$ can be computed, for each component $\theta_{j}$ of the parameter vector $\theta$, solving the following two optimization problems

$$
\begin{aligned}
& \underline{\theta_{j}}=\min _{i=1, \ldots, 2^{p}} \underline{\theta_{j i}} \\
& \overline{\theta_{j}}
\end{aligned}
$$

where

$$
\begin{aligned}
& \underline{\theta_{j i}}=\min _{\theta \in \mathcal{D}_{\theta i}^{\prime}} \theta_{j} \\
& \overline{\theta_{j i}}=\max _{\theta \in \mathcal{D}_{\theta i}^{\prime}} \theta_{j}
\end{aligned}
$$

Computation of bounds (12) and (13) requires the solution of $2^{p}$ linear programming problems given by (14) and $2^{p}$ linear programming problems given by (15) for each component of the parameter vector $\theta$ (see [15] for details). The computed bounds implicitly define the parameter uncertainty intervals

$$
P U I_{j}=\left[\underline{\theta_{j}}, \overline{\theta_{j}}\right] \text {. }
$$

In this paper we are interested in computing parameter uncertainty intervals for linear systems that are a-priori known to be stable. In order to explicitly take into account this prior information, the set of all the parameters that belong to $\mathcal{D}_{\theta}^{\prime}$ and guarantee BIBO stability of the identified system will be considered, that is the set $\mathcal{D}_{\theta}^{*}$ defined as

$$
\mathcal{D}_{\theta}^{*}=\mathcal{D}_{\theta}^{\prime} \cap \mathcal{A}_{\theta}^{S T}
$$


where

$$
\begin{gathered}
\mathcal{A}_{\theta}^{S T}=\left\{\theta \in \mathbb{R}^{p}: A(z, \theta) \neq 0 \forall z \in \mathcal{C},|z| \geq 1\right\} \\
A(z, \theta)=z^{n a}+a_{1} z^{n a-1}+\ldots+a_{n a} .
\end{gathered}
$$

Parameter uncertainty intervals for the stable linear systems are defined as

$$
P U I_{j}^{*}=\left[\underline{\theta_{j}^{*}}, \overline{\theta_{j}^{*}}\right]
$$

where:

$$
\begin{aligned}
\underline{\theta_{j}^{*}} & =\min _{\theta \in \mathcal{D}_{\theta}^{*}} \theta_{j} \\
\overline{\theta_{j}^{*}} & =\max _{\theta \in \mathcal{D}_{\theta}^{*}} \theta_{j}
\end{aligned}
$$

Computation of bounds $\theta_{j}^{*}$ and $\overline{\theta_{j}^{*}}$ through the solution of nonlinear nonconvex optimization problems (21) and (22) will be discussed in Section III where a detailed analysis of the geometric structure of $\mathcal{D}_{\theta}^{*}$ is also provided.

\section{COMPUTATION OF THE PARAMETER UNCERTAINTY INTERVALS $P U I_{j}^{*}$}

In this section the mathematical structure of the nonconvex set $\mathcal{D}_{\theta}^{*}$ is analyzed, then it is shown how LMI relaxation techniques can be used to compute parameter bounds $\underline{\theta_{j}^{*}}$ and $\overline{\theta_{j}^{*}}$.

\section{A. Analysis of the mathematical structure of the set $\mathcal{D}_{\theta}^{*}$}

A necessary and sufficient condition for the BIBO stability of the discrete time linear system in Fig. 1 is that the coefficients $a_{1}, \ldots, a_{n a}$ of polynomial $A\left(q^{-1}\right)$ satisfy the Jury's test [16] whose statement is recalled below for self-consistency of the paper.

\section{Jury's test [16]}

The roots of the polynomial $A\left(q^{-1}\right)$ in (2) belong to the unit circle if and only if all the following constraints are satisfied:

$$
\begin{aligned}
& A(1)>0 \\
& (-1)^{n a} A(-1)>0 \\
& \left|a_{n a}\right|<1 \\
& \left|c_{n a-1}\right|<\left|c_{0}\right| \\
& \left|d_{n a-2}\right|<\left|d_{0}\right| \\
& \vdots \\
& \left|q_{2}\right|<\left|q_{0}\right|
\end{aligned}
$$

where $c_{0}, d_{0}, \ldots, q_{0}, \ldots, c_{n a-1}, d_{n a-2}, \ldots, q_{2}, q_{0}$ are polynomial functions of the parameters $a_{1}, a_{2}, \ldots, a_{n a}$, obtained by forming the Jury's array reported in Table I,
Table I. Jury's array.

\begin{tabular}{ccclccc}
\hline$a_{n a}$ & $a_{n a-1}$ & $a_{n a-2}$ & $\ldots$ & $a_{2}$ & $a_{1}$ & 1 \\
1 & $a_{1}$ & $a_{2}$ & $\ldots$ & $a_{n a-2}$ & $a_{n a-1}$ & $a_{n a}$ \\
$c_{n a-1}$ & $c_{n a-2}$ & $c_{n a-3}$ & $\ldots$ & $c_{1}$ & $c_{0}$ & \\
$c_{0}$ & $c_{1}$ & $c_{2}$ & $\ldots$ & $c_{n a-2}$ & $c_{n a-1}$ & \\
$d_{n a-2}$ & $d_{n a-3}$ & $d_{n a-4}$ & $\ldots$ & $d_{0}$ & & \\
$d_{0}$ & $d_{1}$ & $d_{2}$ & $\ldots$ & $d_{n a-2}$ & & \\
$\vdots$ & $\vdots$ & $\vdots$ & $\vdots$ & & & \\
$q_{2}$ & $q_{1}$ & $q_{0}$ & & & & \\
\hline
\end{tabular}

where

$$
\begin{aligned}
c_{n a-j_{c}} & =\operatorname{det}\left(\left[\begin{array}{cc}
a_{n a} & a_{n a-j_{c}} \\
1 & a_{j_{c}}
\end{array}\right]\right), \\
\text { for } j_{c} & =1, \ldots, n a \text { and } a_{0}=1 \\
d_{n a-j_{d}} & =\operatorname{det}\left(\left[\begin{array}{cc}
c_{n a-1} & c_{n a-j_{d}} \\
c_{0} & c_{j_{d}-1}
\end{array}\right]\right),
\end{aligned}
$$$$
\text { for } j_{d}=2, \ldots, n a
$$

$\operatorname{det}(\cdot)$ is the determinant of a matrix and $q_{2}, q_{1}$ and $q_{0}$ are the last three elements of the Jury's array. Therefore, on the basis of the Jury's criterion, the set $\mathcal{A}_{\theta}^{S T}$ can be described as the set of all the parameters values $\theta$ that satisfy the Jury's test. Topological features of the set $\mathcal{A}_{\theta}^{S T}$ are summarized in the following result.

Result 1: If $n a \geq 2, \mathcal{A}_{\theta}^{S T}$ is the union of $2^{n a-2}$ semialgebraic sets, that is

$$
\mathcal{A}_{\theta}^{S T}=\bigcup_{k=1}^{2^{n a-2}} \mathcal{A}_{\theta k}^{S T}
$$

where $\mathcal{A}_{\theta k}^{S T}$ is a semialgebraic set defined by:

- 4 linear inequalities,

- $3(n a-2)$ polynomial inequalities.

Proof - First, note that $\mathcal{A}_{\theta}^{S T}$, defined by inequalities (23) - (28), can be written as:

$$
\mathcal{A}_{\theta}^{S T}=\mathcal{A}_{1} \cap \underbrace{\mathcal{C} \cap \mathcal{D} \cap \ldots \cap \mathcal{Q}}_{\text {intersection of } n a-2}
$$

where:

$$
\begin{aligned}
\mathcal{A}_{1}=\left\{\theta \in \mathbb{R}^{p}: A(1)>0,\right. & (-1)^{n a} A(-1)>0, \\
\left.\left|a_{n a}\right|<1\right\} & \\
\mathcal{C} & =\left\{\theta \in \mathbb{R}^{p}:\left|c_{n a-1}\right|<\left|c_{0}\right|\right\} \\
\mathcal{D} & =\left\{\theta \in \mathbb{R}^{p}:\left|d_{n a-2}\right|<\left|d_{0}\right|\right\} \\
\vdots & \\
\mathcal{Q} & =\left\{\theta \in \mathbb{R}^{p}:\left|q_{2}\right|<\left|q_{0}\right|\right\}
\end{aligned}
$$

Besides, $\mathcal{C}=\mathcal{C}_{1} \cup \mathcal{C}_{2}, \mathcal{D}=\mathcal{D}_{1} \cup \mathcal{D}_{2}$ and so on, up to 
$\mathcal{Q}=\mathcal{Q}_{1} \cup \mathcal{Q}_{2}$, where:

$$
\begin{aligned}
& \mathcal{C}_{1}=\left\{\theta \in \mathbb{R}^{p}: \quad c_{0} \geq 0,-c_{0} \leq c_{n a-1} \leq c_{0}\right\} \\
& \mathcal{C}_{2}=\left\{\theta \in \mathbb{R}^{p}: \quad c_{0}<0, c_{0} \leq c_{n a-1} \leq-c_{0}\right\} \\
& \mathcal{D}_{1}=\left\{\theta \in \mathbb{R}^{p}: \quad d_{0} \geq 0,-d_{0} \leq d_{n a-2} \leq d_{0}\right\} \\
& \mathcal{D}_{2}=\left\{\theta \in \mathbb{R}^{p}: \quad d_{0}<0, d_{0} \leq d_{n a-2} \leq-d_{0}\right\} \\
& \mathcal{Q}_{1}=\left\{\theta \in \mathbb{R}^{p}: \quad q_{0} \geq 0,-q_{0} \leq q_{2} \leq q_{0}\right\} \\
& \mathcal{Q}_{2}=\left\{\theta \in \mathbb{R}^{p}: q_{0}<0, q_{0} \leq q_{2} \leq-q_{0}\right\}
\end{aligned}
$$

Therefore, Eq. (32) can be rewritten as

$$
\begin{aligned}
\mathcal{A}_{\theta}^{S T}=\mathcal{A}_{1} \cap\left(\mathcal{C}_{1} \cup \mathcal{C}_{2}\right) \cap\left(\mathcal{D}_{1} \cup \mathcal{D}_{2}\right) \ldots \cap\left(\mathcal{Q}_{1} \cup \mathcal{Q}_{2}\right) \\
=\underbrace{\left(\mathcal{A}_{1} \cap \mathcal{C}_{1} \cap \mathcal{D}_{1} \ldots \cap \mathcal{Q}_{1}\right)}_{\mathcal{A}_{\theta 1}^{S T}} \cup \underbrace{\left(\mathcal{A}_{1} \cap \mathcal{C}_{1} \cap \mathcal{D}_{1} \ldots \cap \mathcal{Q}_{2}\right)}_{\mathcal{A}_{\theta 2}^{S T}} \cup \\
\\
\ldots \cup \underbrace{\left(\mathcal{A}_{1} \cap \mathcal{C}_{2} \cap \mathcal{D}_{2} \cap \ldots \cap \mathcal{Q}_{2}\right)}_{\mathcal{A}_{\theta 2^{\text {na }}}^{\text {( }}}
\end{aligned}
$$

Each set $\mathcal{A}_{k}^{S T}$, for $k=1, \ldots, 2^{n a-2}$, is then given by the intersection of $\mathcal{A}_{1}, \mathcal{C}_{c}, \mathcal{D}_{d}, \ldots, \mathcal{Q}_{q}$, for all possible combination of the index $c=1,2, d=1,2, \ldots, q=1,2$. Since $\mathcal{A}_{1}$ in (33) is defined by 4 linear inequalities and each one of the sets $\mathcal{C}_{1}, \mathcal{C}_{2}, \mathcal{D}_{1}, \mathcal{D}_{2}, \ldots, \mathcal{Q}_{1}, \mathcal{Q}_{2}$ described by equations (37) - (42) is defined by 3 polynomial inequalities, $\mathcal{A}_{\theta k}^{S T}$ results to be a semialgebraic set.

Remark 1: For the case $n a \leq 2$, it is not necessary to form the Jury's array. As a matter of fact, when $n a=1$, the root of the polynomial $A\left(q^{-1}\right)$ has modulus less than 1 if and only if the linear inequalities (23) and (24) are satisfied. Thus, when $n a=1, \mathcal{A}_{\theta}^{S T}$ is a convex set defined by 2 linear constraints. Analogously, when $n a=2$, the root of the polynomial $A\left(q^{-1}\right)$ has modulus less than 1 if and only if the linear inequalities (23) - (25) are satisfied. In this case, $\mathcal{A}_{\theta}^{S T}$ results to be a convex set defined by 4 linear constraints.

The next result provides a description of the geometrical structure of $\mathcal{D}_{\theta}^{*}$. In order to comply with the conference page limit constraint, the proof of all the following results are omitted. The interested reader is referred to [17].

Result 2: $\mathcal{D}_{\theta}^{*}$ is the union of $2^{2 n a+n b-1}$ semialgebraic sets $\mathcal{D}_{\theta i k}^{*}$, that is:

$$
\mathcal{D}_{\theta}^{*}=\bigcup_{i=1}^{2^{p}} \bigcup_{k=1}^{2^{n a-2}} \mathcal{D}_{\theta i k}^{*}
$$

where

$$
\mathcal{D}_{\theta i k}^{*}=\mathcal{D}_{\theta i}^{\prime} \cap \mathcal{A}_{k}^{S T}
$$

for all $i=1, \ldots, 2^{p}$ and $k=1, \ldots, 2^{n a-2}$. Besides, each set $\mathcal{D}_{\theta i k}^{*}$ is defined by:

- $p+2 N$ linear inequalities that define $\mathcal{D}_{\theta i}^{\prime}$,

- 4 linear inequalities $+3(n a-2)$ polynomial inequalities that define $\mathcal{A}_{\theta k}^{S T}$

\section{B. Computation of PUI* by means of LMI relaxation tech-} niques

In this section a procedure to compute approximate solutions of problems (21) and (22) is discussed.

Result 3: Bounds $\theta_{j}^{*}$ and $\overline{\theta_{j}^{*}}$ can be computed solving the following optimization problems:

$$
\underline{\theta_{j}^{*}}=\min _{\substack{i=1, \ldots, 2^{p} \\ k=1, \ldots, 2^{\text {na-2 }}}} \underline{\theta_{j i k}^{*}}
$$

where

$$
\begin{aligned}
& \underline{\theta_{j i k}^{*}}=\min _{\theta \in \mathcal{D}_{\theta i k}^{*}} \theta_{j} \\
& \overline{\theta_{j i k}^{*}}=\max _{\theta \in \mathcal{D}_{\theta i k}^{*}} \theta_{j}
\end{aligned}
$$

Results 2 and 3 show that the evaluation of the parameter uncertainty interval $P U I_{j}^{*}$ for all the components of the parameter vector $\theta$ requires, in the general case, the solutions of $2 p 2^{2 n a+n b-1}$ semialgebraic optimization problems with $p$ optimization variables and $m=p+2 N+4+3(n a-2)=4 n a+n b+2 N-2$ constraints. However, in many practical situations, $\mathcal{D}_{\theta}^{\prime}$ lies only in few orthants of the parameter space $\mathbb{R}^{p}$ which means that a large number of subset $\mathcal{D}_{\theta i}^{\prime}$ results to be empty. When such a case occurs, the number of optimization problems to be solved can be significantly reduced since the number of subsets $\mathcal{D}_{\theta i k}^{*} \neq \emptyset$ is small. Thus, in order to reduce the computational complexity of the proposed approach, we suggest first to compute the $P U I_{j}$ for all $j=1, \ldots, p$. Such a computation can be performed by means of linear programming (LP) techniques. Analysis of the signs of bounds $\theta_{j}$ and $\overline{\theta_{j}}$ allow us to detect which orthants are not intersected by the feasible set $\mathcal{D}_{\theta}^{\prime}$. Then, (47) and (48) can be solved by constraining the index $i$ to belong to the set $\mathcal{I}_{\mathcal{D}_{\theta}^{\prime}}=\left\{i=1, \ldots, 2^{p}: \quad \mathcal{D}_{\theta i}^{\prime} \neq \emptyset\right\}$.

Considerable efforts have been devoted in the last years to approximate semialgebraic optimization problems by a hierarchy of convex LMI relaxations (see the survey paper [18] for a review of the literature on the subject). In particular, the approach proposed in [19] is based on the representation of nonnegative polynomials as Sum of Squares (SOS), while in [20] the dual theory of moments is exploited. More specifically, the relaxation technique described in [20] solves semidefinite programming (SDP) problems, whose optima converge to the global optima of the original problem as the length of the number of successive LMI relaxations, the so called relaxation order $\delta$, increases. An efficient MATLAB implementation of this relaxation technique has been developed in the open source software Gloptipoly [21] which exploits the SDP solver SeDuMi [22] to solve optimization problems in polynomial time. In this paper, the method presented in [20] is applied to relax (21) and (22) 
to convex optimization problems, leading to the computation of the $\delta$-relaxed parameter uncertainty intervals defined as:

$$
P U I_{j}^{*}(\delta)=\left[\underline{\theta_{j}^{*}}(\delta), \overline{\theta_{j}^{*}}(\delta)\right], j=1, \ldots, n
$$

where $\theta_{j}^{*}(\delta)$ and $\overline{\theta_{j}^{*}}(\delta)$ are optimal solutions of the SDP problem obtained by applying the theory of moments for a relaxation order $\delta$ to (21) and (22) respectively.

Remark 2: If $n a \leq 2, \mathcal{D}_{\theta}^{*}$ is defined by a set of linear inequalities (as pointed out in Remark 1). Therefore, global optima of problems (21) and (22) can be computed, in this case, by means of linear programming techniques.

Remark 3: Since constraints described in equations (23) - (28) are strict inequalities, the feasible region $\mathcal{D}_{\theta}^{*}$ is not guaranteed to be a closed set. As a consequence, solutions to problems (21)-(22) are not guaranteed to exist. A possible way to overcome such a technical problem is to modified constraints $(23)-(28)$ as follows:

$$
\begin{aligned}
& A(1) \geq \epsilon \\
& (-1)^{n a} A(-1) \geq \epsilon \\
& \left|a_{n a}\right| \leq 1-\epsilon \\
& \left|c_{n a-1}\right| \leq\left|c_{0}\right|-\epsilon \\
& \left|d_{n a-2}\right| \leq\left|d_{0}\right|-\epsilon \\
& \vdots \\
& \left|q_{2}\right| \leq\left|q_{0}\right|-\epsilon
\end{aligned}
$$

where $\epsilon>0$ can be chosen arbitrarily small.

Remark 4: The prior information on system stability can be also exploited in the LS estimation by constraining the parameter $\theta$ to belong to $\mathcal{A}_{\theta}^{S T}$. Then, the LS estimation problem with stability constraints can be formulated as

$$
\theta_{L S}^{*}=\arg \min _{\theta \in \mathcal{A}_{\theta}^{S T}} \sum_{t=n a+1}^{N}\left(y_{t}-\theta^{\mathrm{T}} \phi_{t}\right)^{2} .
$$

From Result 1, the nonconvex optimization problem (56) can be written as the collection of $2^{n a-2}$ semialgebraic optimization problems

$$
\begin{gathered}
\theta_{L S, i}^{*}=\arg \min _{\theta \in \mathcal{A}_{\theta i}^{S T}} \sum_{t=n a+1}^{N}\left(y_{t}-\theta^{\mathrm{T}} \phi_{t}\right)^{2} \\
\text { with } i=1, \ldots, 2^{n a-2},
\end{gathered}
$$

whose approximate optimal solutions can be found through the convex LMI relaxation techniques previously described. The optimal LS estimator $\theta_{L S}^{*}$ guaranteed to belong to the region $\mathcal{A}_{\theta}^{S T}$ is then computed by solving the minimization problem over a $2^{n a-2}$-element set, that is

$$
\theta_{L S}^{*}=\arg \min _{\substack{\theta_{L S, i}^{*} \\ i=1, \ldots, 2^{n a-2}}} \sum_{t=n a+1}^{N}\left(y_{t}-\theta_{L S, i}^{*^{\mathrm{T}}} \phi_{t}\right)^{2} .
$$

\section{PROPERTIES OF RELAXED PARAMETER UNCERTAINTY INTERVALS $P U I_{j}^{*}(\delta)$}

The following results present some properties of the relaxed stable parameter uncertainty intervals $P U I_{j}^{*}(\delta)$.

\section{Result 4: Guaranteed relaxed uncertainty intervals.}

For any relaxation order $\delta$, the $\delta$-relaxed parameter uncertainty interval $P U I_{j}^{*}(\delta)$ is guaranteed to contain the true unknown parameter $\theta_{j}$ to be estimated, for all $j=1, \ldots, p$, i.e.

$$
\theta_{j} \in P U I_{j}^{*}(\delta) \text { for all } j=1, \ldots, p \text {. }
$$

\section{Result 5: Convergence to tight parameter uncertainty} interval $P U I_{j}^{*}$.

The $\delta$-relaxed parameter uncertainty interval $P U I_{j}^{*}(\delta)$ converges to the tight parameter uncertainty interval $P U I_{j}^{*}$ as far as the relaxation order goes to infinity, i.e.:

$$
\lim _{\delta \rightarrow \infty} \underline{\theta_{j}^{*}}(\delta)=\underline{\theta_{j}^{*}}, \quad \lim _{\delta \rightarrow \infty} \overline{\theta_{j}^{*}}(\delta)=\overline{\theta_{j}^{*}}
$$

Result 6: Accuracy improvement of $P U I_{j}^{*}$ over $P U I_{j}$. The $\delta$-relaxed stable parameter uncertainty intervals $P U I_{j}^{*}(\delta)$ of equation (49) are included in the $P U I_{j}$ of equation (16) for any value of the relaxation order $\delta$, that is:

$$
P U I_{j}^{*}(\delta) \subseteq P U I_{j}
$$

\section{A simulate EXAMPLE}

In this section a simulated example is presented in order to show the effectiveness of the presented approach. A third order system is considered, characterized by (2) and (3), with $A\left(q^{-1}\right)=\left(1+0.9 q^{-1}-0.85 q^{-2}-0.95 q^{-3}\right)$ and $B\left(q^{-1}\right)=\left(2.27 q^{-1}-1.25 q^{-2}-0.92 q^{-3}\right)$. Thus, the true parameter vector is $\theta^{\mathrm{T}}=\left[\begin{array}{llllll}a_{1} & a_{2} & a_{3} & b_{1} & b_{2} & b_{3}\end{array}\right]=$

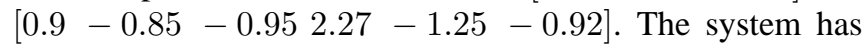
been excited by a random input sequence uniformly distributed in $[-1,+1]$. Both input and output data sequences have been corrupted by random additive uncertainties $\xi_{t}$ and $\eta_{t}$, uniformly distributed in $\left[-\Delta \xi_{t},+\Delta \xi_{t}\right]$ and $\left[-\Delta \eta_{t},+\Delta \eta_{t}\right]$, respectively. The chosen error bounds $\Delta \xi_{t}$ and $\Delta \eta_{t}$ are such that the Signal to Noise Ratios on the input $S N R_{x}$ and on the output $S N R_{w}$, defined as

$$
S N R_{x}=10 \log \left(\frac{\sum_{t=1}^{N} x_{t}^{2}}{\sum_{t=1}^{N} \xi_{t}^{2}}\right), S N R_{w}=10 \log \left(\frac{\sum_{t=1}^{N} w_{t}^{2}}{\sum_{t=1}^{N} \eta_{t}^{2}}\right)
$$

are equal to $33 \mathrm{db}$ and $48 \mathrm{db}$, respectively. The length of the data sequence is $N=300$. First, bounds $\theta_{j}$ and $\overline{\theta_{j}}$ defining $P U I_{j}$ are evaluated without imposing the stability constraints. The obtained results are reported in Table II together with the central estimated $\theta_{j}^{c}$ and the parameter uncertainty $\Delta \theta_{j}$, defined as

$$
\theta_{j}^{c}=\frac{\overline{\theta_{j}}+\underline{\theta_{j}}}{2}, \quad \Delta \theta_{j}=\frac{\overline{\theta_{j}}-\underline{\theta_{j}}}{2}
$$


Then, stability constraints (50) - (55) have been imposed to compute bounds $\theta_{j}^{*}$ and $\overline{\theta_{j}^{*}}$ through the solution of problems (21)-(22) with an LMI relaxation order $\delta=2$. Table III shows the obtained values of $\theta_{j}^{*}$ and $\overline{\theta_{j}^{*}}$, the central estimate $\theta_{j}^{c *}$ and the parameter uncertainty $\Delta \theta_{j}^{*}$, defined as

$$
\theta_{j}^{c *}=\frac{\overline{\theta_{j}^{*}}+\theta_{j}^{*}}{2}, \quad \Delta \theta_{j}^{*}=\frac{\overline{\theta_{j}^{*}}-\underline{\theta_{j}^{*}}}{2}
$$

Comparison between results reported in Table II and Table III shows that, imposition of stability constraints leads to a significant reduction of parameters uncertainty for both the coefficients of the denominator $A\left(q^{-1}\right)$, and the coefficients of numerator $B\left(q^{-1}\right)$, although stability constraints involves polynomial $A\left(q^{-1}\right)$ only. The improvement on the estimation accuracy is particulary noticeable for the denominator parameters $a_{1}, a_{2}$ and $a_{3}$ as shown by the value of $\Delta \theta_{j}^{*}$ which, for each $j=1,2,3$, is at least $50 \%$ less than $\Delta \theta_{j}$.

Table II: Parameter central estimates $\left(\theta_{j}^{c}\right)$, parameter bounds $\left(\theta_{j}, \overline{\theta_{j}}\right)$ and parameter uncertainty bounds $\Delta \theta_{j}$ (without stability constraints).

\begin{tabular}{cccccc}
\hline Parameter & $\begin{array}{c}\text { True } \\
\text { Value }\end{array}$ & $\underline{\theta_{j}}$ & $\theta_{j}^{c}$ & $\overline{\theta_{j}}$ & $\Delta \theta_{j}$ \\
\hline$a_{1}$ & 0.9000 & 0.3904 & 0.7987 & 1.2070 & 0.4083 \\
$a_{2}$ & -0.8500 & -1.7604 & -1.0349 & -0.3093 & 0.7255 \\
$a_{3}$ & -0.9500 & -1.4561 & -1.0514 & -0.6467 & 0.4047 \\
$b_{1}$ & 2.2700 & 1.5388 & 2.3212 & 3.1036 & 0.7824 \\
$b_{2}$ & -1.2500 & -2.3156 & -1.3592 & -.4027 & 0.9565 \\
$b_{3}$ & -0.9200 & -1.7957 & -0.9802 & -0.1647 & 0.8155 \\
\hline
\end{tabular}

Table III: Parameter central estimates $\left(\theta_{j}^{c *}\right)$, parameter bounds $\left(\theta_{j}^{*}, \overline{\theta_{j}^{*}}\right)$ and stable parameter uncertainty bounds $\Delta \theta_{j}^{*}$ (with stability constraints).

\begin{tabular}{cccccc}
\hline Parameter & $\begin{array}{c}\text { True } \\
\text { Value }\end{array}$ & $\underline{\theta_{j}^{*}}$ & $\theta_{j}^{\text {c* }}$ & $\overline{\theta_{j}^{*}}$ & $\Delta \theta_{j}^{*}$ \\
\hline$a_{1}$ & 0.9000 & 0.8251 & 1.0104 & 1.1956 & 0.1853 \\
$a_{2}$ & -0.8500 & -0.9127 & -0.6110 & -0.3093 & 0.3017 \\
$a_{3}$ & -0.9500 & -1.000 & -0.8234 & -0.6467 & 0.1766 \\
$b_{1}$ & 2.2700 & 1.5388 & 2.3193 & 3.0998 & 0.7805 \\
$b_{2}$ & -1.2500 & -2.1455 & -1.2741 & -0.4027 & 0.8714 \\
$b_{3}$ & -0.9200 & -1.7584 & -0.9616 & -0.1647 & 0.7969 \\
\hline
\end{tabular}

\section{CONCLUDING REMARKS AND FUTURE WORKS}

Set-membership identification of linear systems a priori known to be stable is addressed in the paper. First, it is shown that explicit enforcement of stability constraints in the evaluation of parameter bounds leads to complex nonconvex optimization problems. Then, suitable relaxation techniques are presented to compute global optima of those problems. The computed relaxed bounds are shown to converge monotonically to the global solution of the original nonconvex problems as far as the relaxation order goes to infinity. Furthermore, accuracy improvement over the parameter bounds computed without stability constraints, irrespective of the value of the relaxation order, is theoretically proved. Effectiveness of the proposed technique is shown by means of a simulated example.

The convex relaxation approach discussed in the paper is based on a detailed analysis of the geometrical structure of the mathematical constraints arising from the necessary and sufficient stability conditions provided by the Jury's test. Therefore, the idea presented in the paper can readily be applied also outside the Set-membership framework. For instance, the computation of Least squares estimate, constrained to the set of parameters satisfying the Jury's test conditions, requires the solution of a finite number of semialgebraic problems.

\section{REFERENCES}

[1] L. Ljung, System Identification, Theory for the User. Upper Saddle River: Prentince Hall, 1999.

[2] T. Söderström and P. Stoica, "On the stability of dynamic models obtained by least-squares identification," IEEE Trans. Automatic Control, vol. 26, no. 2, pp. 575-577, 1981.

[3] P. Regalia and P. Stoica, "Stability of multivariable least-squares models," IEEE Signal. Processing Letters, vol. 2, no. 10, pp. 195196, 1995.

[4] J. Tugnait and C. Tontiruttananon, "Identification of linear systems via spectral analysis given time-domain data: Consistency, reduced-order approximation, and performance analysis," IEEE Trans. Automatic Control, vol. 43, no. 10, pp. 1354-1373, 1998.

[5] P. Janakiraman and A. Bhattacharyya, "Stable output error identification scheme using periodic excitation signals," in Proc. of IEEE International Symposium on Circuits and Systems, vol. 5, 1999, pp. 539-542.

[6] G. D. Nicolao and G. Pillonetto, "A new kernel-based approach for system identification," in Proc. of the American Control Conference, 2008, pp. 4510-4516.

[7] S. Lacy and D. Bernstein, "Subspace identification with guaranteed stability using constrained optimization," IEEE Trans. Automatic Control, vol. 48, no. 7, pp. 1259-1263, 2003.

[8] M. Milanese and A. Vicino, "Optimal estimation theory for dynamic sistems with set membership uncertainty: an overview," Automatica, vol. 27(6), pp. 997-1009, 1991.

[9] E. Walter and H. Piet-Lahanier, "Estimation of parameter bounds from bounded-error data: a survey," Mathematics and Computers in simulation, vol. 32, pp. 449-468, 1990.

[10] M. Milanese, J. Norton, H. Piet-Lahanier, and E. Walter, Eds., Bounding approaches to system identification. New York: Plenum Press, 1996.

[11] J. Norton (Ed.), "Special issue on bounded-error estimation," Int. J. of Adapt. Control \& Sign. Proces., vol. 8, no. 1, 1994.

[12] - "Special issue on bounded-error estimation," Int. J. of Adapt. Control \& Sign. Proces., vol. 9, no. 1, 1995.

[13] S. Veres and J. Norton, "Parameter-bounding algorithms for linear errors in variables models," in Proc. of IFAC/IFORS Symposium on Identification and System Parameter Estimation, 1991, pp. 1038-1043.

[14] V. Cerone, "Parameter bounds for armax models from records with bounded errors in variables," Int. J. Control, vol. 57, no. 1, pp. 225235, 1993.

[15] — , "Feasible parameter set for linear models with bounded errors in all variable," Automatica, vol. 29, no. 6, pp. 1551-1555, 1993.

[16] E. Jury, The theory and application of Z-transform method. New York: Wiley, 1964.

[17] V. Cerone, D. Piga, and D. Regruto, "Bounding the parameters of linear systems with stability constraints," DAUIN Internal Report 2010/03/10, 2010.

[18] M. Laurent, "Sums of squares, moment matrices and optimization over polynomials," Emerging Applications of Algebraic Geometry, Vol. 149 of IMA Volumes in Mathematics and its Applications, M. Putinar and S. Sullivant (eds.), pp. 157-270, 2009.

[19] P. Parrillo, "Semidefinite programming relaxations for semialgebraic problems," Mathematical Programming, vol. 96, pp. 293-320, 2003.

[20] J. B. Lasserre, "Global optimization with polynomials and the problem of moments," SIAM Journal on Optimization, vol. 11, pp. 796-817, 2001.

[21] D. Henrion and J. B. Lasserre, "Gloptipoly: Global optimization over polynomials with Matlab and SeDuMi," ACM Transactions Math. Soft, vol. 29, pp. 165-194, 2003.

[22] J. F. Sturm, "Using SeDuMi 1.02, a MATLAB Toolbox for optimization over symmetric cones," Optim. Methods Software, vol. 11, no. 12, pp. 625-653, 1999. 